\title{
Experimental test of the first- and second-order duality relations for the two-photon states
}

\author{
Jie-Hui Huang, ${ }^{1,3}$ Hong-Yu Liu, ${ }^{2}$ Jiang-Rui Gao, ${ }^{2}$ M. Suhail Zubairy, ${ }^{1,4}$ and Shi-Yao Zhu ${ }^{1,2}$ \\ ${ }^{1}$ Beijing Computational Science Research Center, Beijing 100084, People's Republic of China \\ ${ }^{2}$ The State Key Laboratory of Quantum Optics and Quantum Optics Devices, Institute of Opto-Electronics, Shanxi University, \\ Taiyuan 030006, People's Republic of China \\ ${ }^{3}$ College of Physics and Communication Electronics, Jiangxi Normal University, Nanchang 330022, People's Republic of China \\ ${ }^{4}$ Institute of Quantum Science and Engineering (IQSE) and Department of Physics and Astronomy, Texas A\&M University, \\ College Station, Texas 77843-4242, USA
}

(Received 21 February 2013; published 19 July 2013)

\begin{abstract}
The first- and second-order wave-particle dualities for two-photon states are investigated in a Mach-Zehnder interferometer. These duality relations are experimentally tested through two-photon pure states and two-photon mixed states. The experimental results are in full agreement with the theoretical predictions. The optical fields exhibiting the same first-order particlelike and wavelike behaviors may carry entirely different second-order duality information, which implies that the higher-order duality relations are a useful tool for analyzing the wave-particle duality for multiphoton states.
\end{abstract}

DOI: 10.1103/PhysRevA.88.013828

PACS number(s): 42.50.Xa, 42.50.Ct, 03.65.Ta, 42.50.Ar

\section{INTRODUCTION}

In 1927, Bohr introduced the important principle of complementarity in quantum mechanics [1] which predicts equally real but mutually exclusive properties of quantum systems [2,3]. The wave-particle duality is the most important example of complementarity which tells us that the wavelike and particlelike behaviors of quantum objects are mutually exclusive. The earlier discussion on complementarity was based on Young's double-slit experiment with a light beam that contained many photons [4,5]. In the 1980s, an experimentally realizable duality was put forward based on a two-path Mach-Zehnder interferometer fed with single photons. Two quantities, the visibility of the interference pattern behind the interferometer, $V$, and the distinguishability of the photon passing along the two paths, $D$, are introduced to measure the wavelike and particlelike behaviors of the photon, respectively, and the duality of single photons can then be mathematically described by the inequality [6-9], $D^{2}+V^{2} \leqslant 1$, where the equality sign holds for single-photon pure states. For the single-photon mixed states, the lost duality information causes the increase of quantum uncertainty [10]. This duality relation has been verified in several experiments [11], even when the choice of measuring schemes is delayed after the single photon's entrance into the interferometer [12]. The experimental delayed-choice test of the above duality relation at the single-photon level was first reported in 2007 and 2008 [13,14]. Recently, by introducing additional degrees of the single photon (its polarization), a new optical device named the quantum beam splitter (QBS) is proposed in Refs. [15,16], and is experimentally realized in different ways [17-19]. With the additional degree in the QBS, the above duality relation is still valid $[18,19]$. Note that the theory and experiments are all based on the single particle (photon).

However, the duality at a single-particle level is not the whole story about the duality. How to describe the duality relation for a light beam still lies ahead of us. That is to say, we need to consider the full particlelike and wavelike behaviors of multiphotons. Recently, we have introduced two quantities to define high-order visibility and distinguishability [20], which generalizes the discussion of the duality from a single-photon case to multiphoton cases. For the $N$-photon component of a quantum state, there are $N$ duality relations, which can provide us more complete information about the particlelike and wavelike behaviors of the multiphotons than any single duality relation. This helps us to have a deep insight on duality for a light beam composed of more than one photon.

The organization of the paper is as follows. In Sec. II we briefly review the concept of high-order duality, and present some theoretical results on the duality of two-photon pure or mixed states. In Sec. III, we present results of the experimental measurement of the first- and second-order dualities for the two-photon pure and mixed states. In Sec. IV, we compare the experimental result for the first- and second-order duality of the two-photon pairs with the theoretical prediction. In Sec. V, we present our conclusions.

\section{HIGHER-ORDER WAVE-PARTICLE DUALITY}

In order to study the duality of multiphotons, we introduced the concepts of the $k$ th-order distinguishability and the $k$ thorder fringe visibility in a recent work [20]. The $k$ th-order distinguishability, defined in terms of the Hermitian operator [21],

$$
\hat{D}_{k} \equiv \frac{\left(a_{1}^{+}\right)^{k} a_{1}^{k}-\left(a_{2}^{+}\right)^{k} a_{2}^{k}}{\left\langle\left(a_{1}^{+}\right)^{k} a_{1}^{k}\right\rangle+\left\langle\left(a_{2}^{+}\right)^{k} a_{2}^{k}\right\rangle}
$$

is to quantify the $k$ th-order particlelike behavior of multiphotons in a Mach-Zehnder interferometer; see Fig. 1. Here $a_{1}^{+}$ $\left(a_{1}\right)$ and $a_{2}^{+}\left(a_{2}\right)$ denote the creation (annihilation) operators of the modes in paths 1 and 2 in the interferometer, and the denominator $\left\langle\left(a_{1}^{+}\right)^{k} a_{1}^{k}\right\rangle+\left\langle\left(a_{2}^{+}\right)^{k} a_{2}^{k}\right\rangle$ is for normalization. The $k$ th-order wavelike behaviors of multiphotons in the same interferometer are measured through the $k$ th-order fringe visibility,

$$
\hat{V}_{k} \equiv \frac{\left(a_{1}^{+}\right)^{k} a_{2}^{k} e^{i \phi}+\left(a_{2}^{+}\right)^{k} a_{1}^{k} e^{-i \phi}}{\left\langle\left(a_{1}^{+}\right)^{k} a_{1}^{k}\right\rangle+\left\langle\left(a_{2}^{+}\right)^{k} a_{2}^{k}\right\rangle}
$$




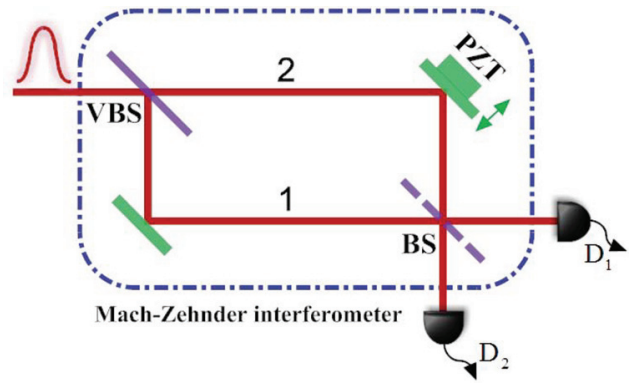

FIG. 1. (Color online) Mach-Zehnder interferometer in a duality experiment. VBS is a variable beam splitter with adjustable reflectivity, PZT is a phase shifter, and BS is a 50:50 beam splitter.

where $\phi$ is the phase difference, which can be varied to maximize or minimize the value of $\left|\left\langle\hat{V}_{k}\right\rangle\right|$. In these definitions, we have used the $k$ th-order autocorrelation $\left(a_{i}^{+}\right)^{k} a_{i}^{k}(i=1,2)$ and the $k$ th-order coherence $\left(a_{1}^{+}\right)^{k} a_{2}^{k}$ to replace the first-order autocorrelation and the first-order coherence used for the traditional distinguishability and visibility, which can now be regarded as the special case of the definitions in (1) by setting $k=1$. The $k$ th-order particle information and the $k$ th-order wave information are just the modules of the corresponding expectation values, i.e., $D_{k}=\left|\left\langle\hat{D}_{k}\right\rangle\right|$ and $V_{k}=\left|\left\langle\hat{V}_{k}\right\rangle\right|_{\max }$.

The physical understanding of the higher-order distinguishability and visibility can be obtained as follows. In view of the identity $\left(a^{+}\right)^{k} a^{k}=\prod_{i=0}^{k-1}\left(a^{+} a-i\right)$, the $k$ thorder distinguishability $D_{k}$ is just the normalized difference between the combination numbers of picking out $k$ photons in path 1 and path 2 . For example, the second-order distinguishability of a quantum state $\alpha\left|n_{1} m_{2}\right\rangle+\beta\left|k_{1} l_{2}\right\rangle$, where the basis $\left|n_{1} m_{2}\right\rangle\left(\left|k_{1} l_{2}\right\rangle\right)$ indicates $n(k)$ photons in path 1 and $m(l)$ photons in path 2 , can be directly calculated as $D_{2}=\left|\frac{C_{1}^{(2)}-C_{2}^{(2)}}{C_{1}^{(2)}+C_{2}^{(2)}}\right|$, where $C_{1}^{(2)}=|\alpha|^{2} n(n-1)+|\beta|^{2} k(k-$ 1) and $C_{2}^{(2)}=|\alpha|^{2} m(m-1)+|\beta|^{2} l(l-1)$ are the average combination number of picking out two photons in path 1 and path 2 in the above state, respectively. Thus the $k$ thorder distinguishability $D_{k}$ can be regarded as the degree of asymmetry of the photon distribution in the two paths. The visibility is determined by the off-diagonal elements of the density matrix in a Fock state basis. For example, the off-diagonal elements $\alpha \beta^{*}\left|n_{1} m_{2}\right\rangle\left\langle k_{1} l_{2}\right|$ and $\alpha^{*} \beta\left|k_{1} l_{2}\right\rangle\left\langle n_{1} m_{2}\right|$ of the above state contribute to the $k$ th-order visibility $V_{k}$ when $k=n-k=l-m$ or $k=k-n=m-l$. The diagonal elements play no role in the evaluation of the visibility.

Our theoretical analysis showed that for the $k$ th-order particle information and the $k$ th-order wave information we have the $k$ th-order duality relation [20],

$$
D_{k}^{2}+V_{k}^{2} \leqslant 1,
$$

which is the generalization of the duality relation for singlephoton cases.

A general two-photon state in the basis $\left\{\left|2_{1} 0_{2}\right\rangle\right.$, $\left.\left|1_{1} 1_{2}\right\rangle,\left|0_{1} 2_{2}\right\rangle\right\}$ can be described by a $3 \times 3$ density matrix,

$$
\rho=\left(\begin{array}{lll}
\rho_{11} & \rho_{12} & \rho_{13} \\
\rho_{21} & \rho_{22} & \rho_{23} \\
\rho_{31} & \rho_{32} & \rho_{33}
\end{array}\right),
$$

where the basis $\left|n_{1} m_{2}\right\rangle$ indicates $n$ photons in path 1 and $m$ photons in path 2 . By imposing the two operators in Eqs. (1a) and (1b) onto the above state, we obtain the first-order distinguishability and visibility in the two-photon case,

$$
\begin{aligned}
D_{1} & =\left|\rho_{11}-\rho_{33}\right|, \\
V_{1} & =\sqrt{2}\left|\rho_{12}+\rho_{23}\right|,
\end{aligned}
$$

and the second-order distinguishability and visibility,

$$
\begin{aligned}
D_{2} & =\frac{\left|\rho_{11}-\rho_{33}\right|}{\rho_{11}+\rho_{33}}, \\
V_{2} & =\frac{2\left|\rho_{13}\right|}{\rho_{11}+\rho_{33}} .
\end{aligned}
$$

Thus the first- and second-order duality relations for the twophoton case are

$$
D_{1}^{2}+V_{1}^{2}=\left|\rho_{11}-\rho_{33}\right|^{2}+2\left|\rho_{12}+\rho_{23}\right|^{2} \leqslant 1,
$$

and

$$
D_{2}^{2}+V_{2}^{2}=1-\frac{4\left(\rho_{11} \rho_{33}-\left|\rho_{13}\right|^{2}\right)}{\left(\rho_{11}+\rho_{33}\right)^{2}} \leqslant 1 .
$$

For the following group of pure states,

$$
\begin{aligned}
|\psi\rangle= & (1-R)\left|2_{1} 0_{2}\right\rangle+\sqrt{2 R(1-R)} e^{i \phi_{0}}\left|1_{1} 1_{2}\right\rangle \\
& +R e^{2 i \phi_{0}}\left|0_{1} 2_{2}\right\rangle,
\end{aligned}
$$

the equal sign is satisfied in both Eqs. (6a) and (6b). Such a two-photon pure state can be experimentally prepared by feeding the Mach-Zehnder interferometer with the two-photon Fock state. Here the parameter $R$ is the reflectivity of the variable beam splitter (VBS) in Fig. 1, and the phase difference between the two paths, $\phi_{0}$, is induced by the VBS and the length difference between the two paths, whose value does not need to be known. For this particular pure state, the first-order distinguishability and visibility [see Eq. (4)] are $D_{1}=|1-2 R|$ and $V_{1}=2 \sqrt{R(1-R)}$, and the secondorder distinguishability and visibility [see Eq. (5)] are $D_{2}=$ $\frac{|1-2 R|}{(1-R)^{2}+R^{2}}$ and $V_{2}=\frac{2 R(1-R)}{(1-R)^{2}+R^{2}}$, respectively. Finally we have $D_{1}^{2}+V_{1}^{2}=D_{2}^{2}+V_{2}^{2}=1$.

If the phase difference $\phi_{0}$ is randomized, we have completely mixed two-photon state with zero off-diagonal elements,

$$
\rho^{\prime}=\left(\begin{array}{ccc}
(1-R)^{2} & 0 & 0 \\
0 & 2 R(1-R) & 0 \\
0 & 0 & R^{2}
\end{array}\right),
$$

which leads to zero visibility $V_{1}=0$ and $V_{2}=0$. In this case, the first-order distinguishability and the second-order distinguishability are $D_{1}=|1-2 R|$ and $D_{2}=\frac{|1-2 R|}{(1-R)^{2}+R^{2}}$, respectively. For the completely mixed state, we have $D_{1}^{2}+$ $V_{1}^{2}<1$ and $D_{2}^{2}+V_{2}^{2}<1$.

\section{EXPERIMENT}

We now experimentally examine the duality relations, Eqs. (6a) and (6b), for the two-photon case. The experimental setup is shown in Fig. 2. The two-photon state is prepared by collinear type-I spontaneous parametric down-conversion (SPDC). We use the second-harmonic generation from a 


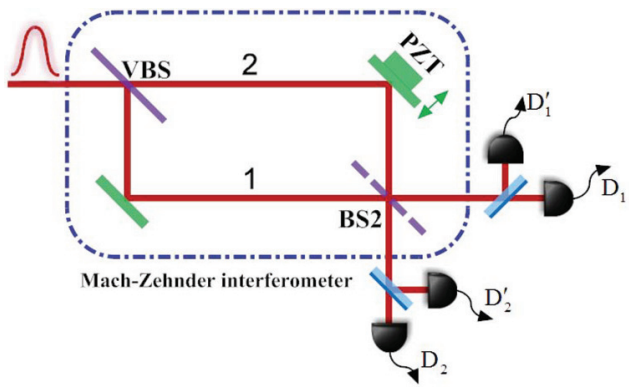

FIG. 2. (Color online) Experimental setup for the input of twophoton pairs: Horizontally polarized photon pairs produced from parametric down-conversion are sent to the interferometer. Each output is equally split by the 50:50 BS, and then connected to two single-photon detectors, $D_{1}$ and $D_{1}^{\prime}\left(D_{2}\right.$ and $\left.D_{2}^{\prime}\right)$.

Ti:sapphire laser at $850 \mathrm{~nm}$ with pulses of width $130 \mathrm{fs}$ and repetition rate $76 \mathrm{MHz}$ to pump a $1 \times 2 \times 0.3-\mathrm{mm}^{3}$ PPKTP (periodically poled $\mathrm{KTiOPO}_{4}$ ) crystal for the SPDC. The pulse generates a two-photon pair at $850 \mathrm{~nm}$ with the same polarization, which is then used as the input of the Mach-Zehnder interferometer. Please note that the vacuum component, which dominates the optical field of the SPDC, has no effect on our data recording. The components with photon number larger than 2 are negligible. Thus the collinear type-I SPDC produces a conditional two-photon Fock state in our experiment, similar to the case of conditional single photons generated through type-II SPDC [22]. After the VBS in Fig. 2, which is composed of a half-wave plate and a polarization beam splitter (PBS), all the photons with horizontal polarization enter path 1 , and the photons with vertical polarization go to path 2 . At the output, BS2 is composed of two PBSs and a half-wave plate in the middle (see Fig. 2). The first PBS is used to combine the two light beams in paths 1 and 2, and the half-wave plate is to rotate the polarization of the two combined light beams by $45^{\circ}$, which overlap and interfere at the second PBS. Finally, we employ four single-photon detectors, $D_{1}, D_{1}^{\prime}, D_{2}$, and $D_{2}^{\prime}$ (see Fig. 2) for the measurement, where the two detectors $D_{1}$ and $D_{1}^{\prime}$ are employed after a 50:50 BS to detect the photons in path 1, and the two detectors $\mathrm{D}_{2}$ and $\mathrm{D}_{2}^{\prime}$, after another 50:50 BS, are for the photons in path 2 . The four single-photon detectors at the output of the interferometer record six coincidence counts, $N_{1,1}, N_{1,2}, N_{1,2^{\prime}}, N_{1^{\prime}, 2}, N_{1^{\prime}, 2^{\prime}}$, and $N_{2,2^{\prime}}$ where the subscripts $1,1^{\prime}, 2$ and $2^{\prime}$ indicate the four single-photon detectors, $D_{1}$, $\mathrm{D}_{1}^{\prime}, \mathrm{D}_{2}$, and $\mathrm{D}_{2}^{\prime}$, respectively. We ignore any event with a single count (single detector click). The two photons can enter the same detector which results in a single click. Since the current photon detectors cannot distinguish two-photon absorption from one-photon absorption, the two-photon pairs being absorbed by the same detector are not counted in our experiment (as only one click). That is to say, $N_{1,1}, N_{1^{\prime}, 1^{\prime}}, N_{2,2}$, and $N_{2^{\prime}, 2^{\prime}}$ are not counted. Now let us analyze the probability of two-photon pairs entering the same detector, $N_{1,1}, N_{1^{\prime}, 1^{\prime}}$, $N_{2,2}$, and $N_{2^{\prime}, 2^{\prime}}$. Any two-photon state $|2\rangle$ (with another input port in the vacuum) is equally split by a 50:50 BS into the superposition of $\frac{1}{\sqrt{2}}\left(\left|2_{1} 0_{2}\right\rangle+\sqrt{2}\left|1_{1} 1_{2}\right\rangle+\left|2_{1} 0_{2}\right\rangle\right)$, which tells us that the total probability for both photons entering the same output path is always equal to the probability for the two photons being separated. Although the sum of the two-photon pairs entering the same detector $D_{1}$ and $D_{1}^{\prime}$ (or $D_{2}$ and $D_{2}^{\prime}$ ) is not counted in our experiment, its contribution to our results is equal to the probability of the coincidence detection by $D_{1}$ and $D_{1}^{\prime}\left(\right.$ or $D_{2}$ and $D_{2}^{\prime}$ ), owing to the 50:50 BS in each output path. That is to say, $N_{1,1}+N_{1^{\prime}, 1^{\prime}}=N_{1^{\prime}, 1}$ and $N_{2,2}+N_{2^{\prime}, 2^{\prime}}=N_{2^{\prime}, 2}$.

For the measurement of the first-order distinguishability $D_{1}$, we need to remove the BS2 in Fig. 2, and record the counting difference between the two paths [see Eq. (1a) with $k=1$ ]. With the same detection efficiency for every detector and the same dissipation rate for all paths, the probability of finding the photons in path $i(i=1,2), p_{i}$, depends on the coincidence counting through the following relation:

$$
\begin{aligned}
& p_{1}=\frac{2\left(N_{1,1}+N_{1^{\prime}, 1^{\prime}}+N_{1,1^{\prime}}\right)+C_{12}}{N}=\frac{4 N_{1,1^{\prime}}+C_{12}}{N}, \\
& p_{2}=\frac{2\left(N_{2,2}+N_{2^{\prime}, 2^{\prime}}+N_{2,2^{\prime}}\right)+C_{12}}{N}=\frac{4 N_{2,2^{\prime}}+C_{12}}{N},
\end{aligned}
$$

with $\quad N=2\left(N_{1,1^{\prime}}+N_{1,1}+N_{1^{\prime}, 1^{\prime}}+N_{2,2^{\prime}}+N_{2,2}+N_{2^{\prime}, 2^{\prime}}\right)+$ $2 C_{12}=4\left(N_{1,1^{\prime}}+N_{2,2^{\prime}}\right)+2 C_{12}$ being the total photon number of the two-photon pairs, and $C_{12}=N_{1,2}+N_{1,2^{\prime}}+N_{1^{\prime}, 2}+$ $N_{1^{\prime}, 2^{\prime}}$ being the total coincidence counting between paths 1 and 2. Note that the two probabilities $p_{1}$ and $p_{2}$ sum up to 1 ; the first-order particle information [see Eq. (1a) with $k=1$ ] can be directly obtained as

$$
D_{1}=\left|p_{1}-p_{2}\right| \text {. }
$$

For the second-order distinguishability $D_{2}$, we also need to remove the BS2 in Fig. 2, and record only two pairs of coincidence counts, $N_{1,1^{\prime}}, N_{2^{\prime}, 2}$, which are actually the second-order autocorrelation of the fields in the two paths 1 and 2 [23], i.e., $\left\langle\left(a_{i}^{+}\right)^{2} a_{i}^{2}\right\rangle \sim N_{i, i^{\prime}}(i=1,2)$. The second-order distinguishability, based on the definition (1a) with $k=2$, turns out to be

$$
D_{2}=\left|\frac{N_{1,1^{\prime}}-N_{2,2^{\prime}}}{N_{1,1^{\prime}}+N_{2,2^{\prime}}}\right| .
$$

For the measurement of the first-order visibility $V_{1}$, we have to employ the BS2 in Fig. 2, and appropriately adjust the phase shifter $\phi$, through the piezoelectric (PZT) in path 2, to maximize the probability $p_{1}$, so that it reaches its maximum value $p_{\max }$, and accordingly, the probability $p_{2}$ reaches its minimum value $p_{\min }$. The difference is just the first-order visibility,

$$
V_{1}=p_{\max }-p_{\min },
$$

which is equivalent to the definition, Eq. (1b), with $k=1$ [20]. Please note the equality $p_{\max }+p_{\min }=1$ always holds for the first-order case. For the measurement of the second-order visibility $V_{2}$, we appropriately adjust the phase shift, $\phi$, through the PZT in path 2, to maximize the sum of the two second-order autocorrelation functions, $\left(N_{1,1^{\prime}}^{\prime}+N_{2^{\prime}, 2}^{\prime}\right)_{\max }$. The sum reaches its minimum values $\left(N_{1,1^{\prime}}^{\prime}+N_{2^{\prime}, 2}^{\prime}\right)_{\min }$ when the phase shift is further retarded by a value of $\pi / 2$, which is realized in experiment through the control of the voltage on the PZT. The second-order visibility is then obtained by

$$
V_{2}=\frac{\left(N_{1,1^{\prime}}^{\prime}+N_{2,2^{\prime}}^{\prime}\right)_{\max }-\left(N_{1,1^{\prime}}^{\prime}+N_{2,2^{\prime}}^{\prime}\right)_{\min }}{N_{1,1^{\prime}}+N_{2,2^{\prime}}},
$$




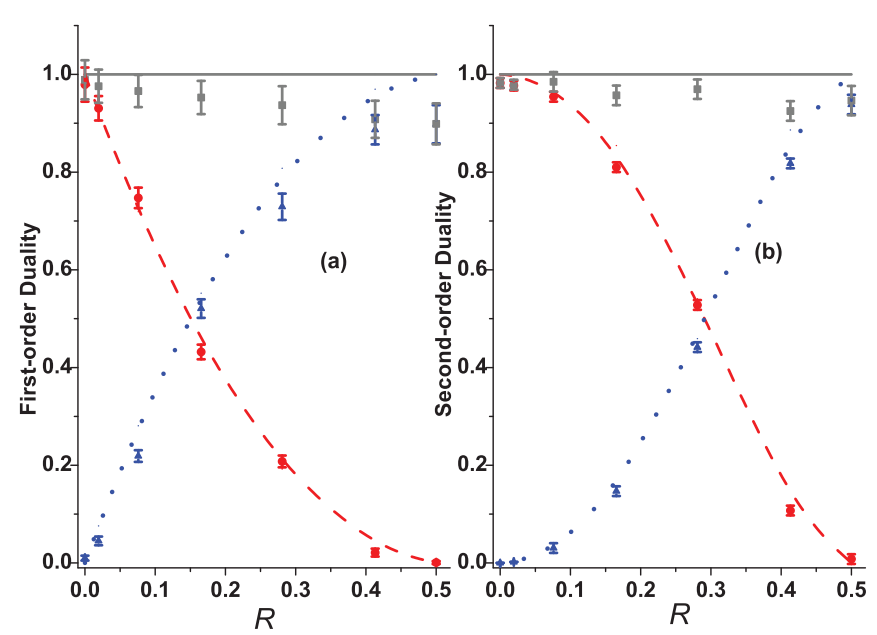

FIG. 3. (Color online) First-order (a) and second-order (b) duality information with the reflectivity of the variable beam splitter $R$ for the pure state (7). In both the first-order and second-order cases, the squared visibility (blue dotted lines) increases with the decrease of the squared distinguishability (red dashed lines), and theoretically keeps their sum (gray solid lines) a constant. Points with error bar are experimental data.

which is equal to the maximum expectation value of Eq. (1b) with $k=2$, over the parameter $\phi$, i.e., $\left|\left\langle\hat{V}_{2}\right\rangle\right|_{\max }$ [20].

If the two-photon flux is prepared in the two-photon pure state (7), the theoretical values of the six coincidence counts among the four single-photon detectors, $\mathrm{D}_{1}, \mathrm{D}_{1}^{\prime}, \mathrm{D}_{2}$, and $\mathrm{D}_{2}^{\prime}$, are $N_{1,1^{\prime}}=\frac{N}{4}(1-R)^{2}, N_{2,2^{\prime}}=\frac{N}{4} R^{2}, N_{1,2^{\prime}}=N_{1,2}=N_{1^{\prime}, 2^{\prime}}=$ $N_{1^{\prime}, 2}=\frac{N}{4} R(1-R)$ for the measurement of the distinguishablity $D_{1}$ and $D_{2}$, and $N_{1,1^{\prime}}^{\prime}=\frac{N^{\prime}}{16}\left|\sqrt{1-R}+\sqrt{R} e^{i\left(\phi_{0}+\phi\right)}\right|^{4}$, $N_{2,2^{\prime}}^{\prime}=\frac{N^{\prime}}{16}\left|\sqrt{1-R}-\sqrt{R} e^{i\left(\phi_{0}+\phi\right)}\right|^{4}, \quad N_{1,2^{\prime}}^{\prime}=N_{1,2}^{\prime}=N_{1^{\prime}, 2^{\prime}}^{\prime}=$ $N_{1^{\prime}, 2}^{\prime}=\frac{N^{\prime}}{16}\left|\operatorname{Re}^{2 i\left(\phi_{0}+\phi\right)}+R-1\right|^{2}$ for the measurement of the visibility $V_{1}$ and $V_{2}$, where $N_{i, j}\left(N_{i, j}^{\prime}\right)$ is the coincidence count in the measurement of the distinguishability (visibility). For the measurement of the second-order visibility $V_{2}$, the sum of the two coincidence counts $\left(N_{1,1^{\prime}}^{\prime}+N_{2^{\prime}, 2}^{\prime}\right)$ reaches its maximum value, $\left(N_{1,1^{\prime}}^{\prime}+N_{2^{\prime}, 2}^{\prime}\right)_{\max }=\frac{N^{\prime}}{8}[1+4 R(1-R)]$, if the phase $\phi$ and the phase $\phi_{0}$ in the initial state [Eq. (7)] satisfy $\cos ^{2}\left(\phi_{0}+\phi\right)=1$, and reaches its minimum value, $\left(N_{1,1^{\prime}}^{\prime}+N_{2^{\prime}, 2}^{\prime}\right)_{\min }=\frac{N^{\prime}}{8}$, in the case of $\cos \left(\phi_{0}+\phi\right)=0$. In the experiments, the important phase is $\left(\phi_{0}+\phi\right)$, and the exact values of $\phi_{0}$ and $\phi$ do not matter.

If the two-photon flux is prepared in the two-photon mixed state [Eq. (8)], the theoretical values of the six coincidence counts are $N_{1,1^{\prime}}=\frac{N}{4}(1-R)^{2}, N_{2,2^{\prime}}=\frac{N}{4} R^{2}$, $N_{1,2^{\prime}}=N_{1,2}=N_{1^{\prime}, 2^{\prime}}=N_{1^{\prime}, 2}=\frac{N}{4} R(1-R)$ for the measurement of distinguishablity $D_{1}$ and $D_{2}$. It is found that the coincidence counts for the measurement of distinguishablity are identical for the pure state (7) and the mixed state (8), so the two states will exhibit the same particlelike behavior in principle. For the measurement of the visibility $V_{1}$ and $V_{2}$ for the mixed state (8), the six coincidence counts are $N_{1,1^{\prime}}^{\prime}=N_{2^{\prime}, 2}^{\prime}=\frac{N^{\prime}}{16}[1+2 R(1-R)]$ and $N_{1,2}^{\prime}=$ $N_{1,2^{\prime}}^{\prime}=N_{1^{\prime}, 2}^{\prime}=N_{1^{\prime}, 2^{\prime}}^{\prime}=\frac{N^{\prime}}{16}|1-2 R(1-R)|$ theoretically. All these coincidence counts above are independent of the phase $\phi$ for the completely mixed state (8). The independence

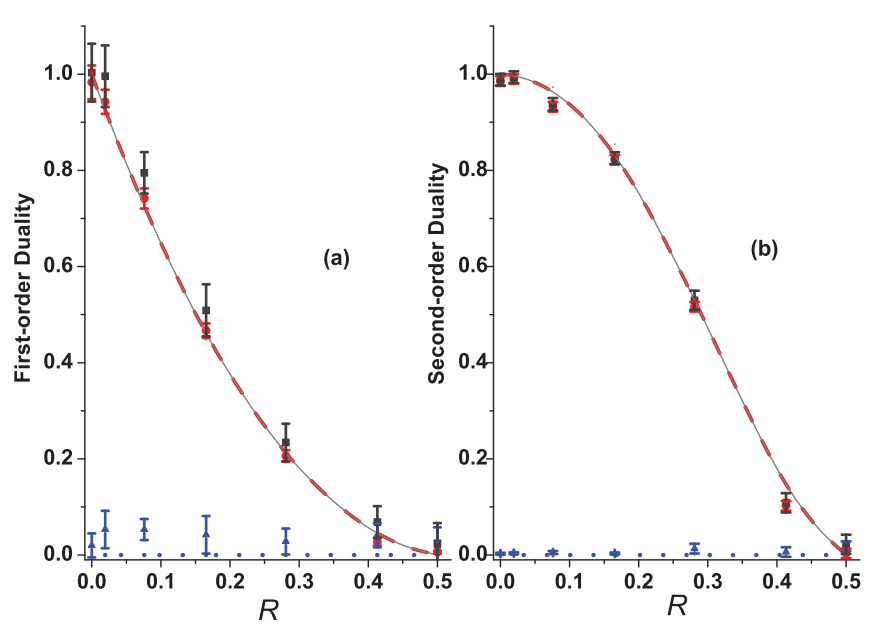

FIG. 4. (Color online) The first-order (a) and second-order (b) duality information with the reflectivity of the variable beam splitter $R$ for the mixed state (8). In both the first-order and second-order cases, the squared visibility (blue dotted lines) theoretically remains zero, and the squared distinguishability (red dashed lines) equals to the sum (gray solid lines) of the two quantities. Points with error bar are experimental data.

relation between the six coincidence counts and the phase $\phi$ can be verified by gradually tilting an optical element in the interferometer, e.g., a PBS, and checking whether the coincidence counts change. Since the PZT plays no role in maximizing or minimizing the required coincidence counts for the completely mixed state, we can use the PZT in our experiment as a part of the preparation of the mixed state (8). In the experiment we randomize the voltage added on the PZT (randomize the phase difference $\phi_{0}$ ) to generate the completed mixed state (in the statistical viewpoint). It follows, on substituting these coincident counts into Eqs. (9)-(11), that we obtain the same results for the distinguishability and visibility as predicted in Eqs. (4) and (5), which are derived based on the operator representation.

The experimental measurements of $D_{1}$ and $D_{2}$ are plotted in Figs. 3(a) and 3(b), as the red circle points with error bar (near the red dashed line). The experimental measurements of $V_{1}$ and $V_{2}$ are plotted in Figs. 3(a) and 3(b), as the blue triangle points with error bar (near the blue dotted line). The sums of $D_{1}^{2}+V_{1}^{2}$ and $D_{2}^{2}+V_{2}^{2}$ are plotted in Figs. 3(a) and 3(b), as the gray square points with error bar (near the gray solid line). It is clear that the sum is almost equal to 1 .

\section{DISCUSSIONS}

The theoretical values for the squared visibility, $V_{1}^{2}$ and $V_{2}^{2}$ (blue dotted lines), and squared distinguishability, $D_{1}^{2}$ and $D_{2}^{2}$ (red dashed lines), and their sums (gray solid lines) are plotted as a function of the reflectivity $R$ in Figs. 3(a) and 3(b), respectively. The points with error bars are the corresponding experimental results. Here we see that the duality relation Eq. (2) with $k=1,2$ is saturated for the two-photon pure states (7). Therefore, the single-photon source is not the only choice to verify the duality relation, even for the first-order duality relation. Note the results for $R=0.5$ to 1.0 are 
the same as $R=0.5$ to 0.0 . In the experiment, the highest visibility is 0.89 , smaller than the theoretical value of 1 due to unbalanced probabilities of the photon entering the two paths of the interferometer, decoherence of the photons, imperfect overlap of the two interfering paths at the output, and unequal detection efficiencies of the detectors.

The duality information for the two-photon mixed state (8) is illustrated in Fig. 4. This state is realized by completely randomizing the phase difference between the two paths in the interferometer. The square of the first-order visibility, $V_{1}^{2}$, and distinguishability, $D_{1}^{2}$, are plotted as blue dotted line and red dashed line, respectively, in Fig. 4(a), and the square of the second-order visibility, $V_{2}^{2}$, and distinguishability, $D_{2}^{2}$, are plotted with the same style in Fig. 4(b). As opposed to the pure-state case discussed above, we obtain almost vanishing visibility for all values of reflectivity $R$, i.e., $V_{1}^{2}=V_{2}^{2}=0$ (see the blue triangle points for the experimental data in Fig. 4). It implies that the interference is not observed for the completely mixed state (8), due to the zero coherence terms (zero off-diagonal elements) in the density matrix (8). With the increasing of the reflectivity $R$, the distinguishability $D_{1}^{2}$ and $D_{2}^{2}$ have the same behavior as for the pure-state case discussed above (see the red circle points for experimental data and red dashed line for the theoretical expectation). These results are in accord with the fact that the visibility for wavelike behavior is determined by the coherence terms in the density matrix, and the distinguishability for particlelike behavior is determined by the diagonal elements in the density matrix as discussed in our theoretical analysis of Eqs. (4) and (5). With the increasing of $R$, the lost particle information does not turn to the wave knowledge of visibility. Thus the duality information, i.e., the total sum of the squared visibility and distinguishability, is not saturated in this mixed-state case (see the gray square points with error bar in Fig. 4).

We can make a comparison between the duality relation of two-photon pairs in the present work and the duality relation of single photons discussed in Ref. [10]. The two-photon pure state (7) and mixed state (8) exhibit the same first-order duality as the single-photon pure state and single-photon mixed state discussed in Ref. [10]. However, as was pointed out in Ref. [20], both the second-order visibility and the second-order distinguishability of a single-photon system are zero, and the second-order duality relation for the two-photon pair input is different from that for the single-photon input. That is to say, two optical fields may exhibit the same first-order duality relation, but different second-order duality relation. The high-order duality relation is important in analyzing the duality experiments for multiphoton sources.

\section{CONCLUSIONS}

We tested experimentally the first- and second-order duality relations for the two-photon states in the Mach-Zehnder interferometer, which is consistent with the theoretical predictions contained in the inequality (2). The first-order duality relation solely is not enough to describe the full duality as the two optical fields exhibiting the same first-order particlelike and wavelike behaviors may have different second-order particlelike and wavelike behaviors. We find that the first-order duality relation is always valid for single-photon states and two-photon states. The sums of the first- and second-order wave information and particle information are equal to 1 for the two-photon pure states, and smaller than 1 for the two-photon mixed states. The higher-order duality may provide us more information about the duality experiments with multiphoton input, and accordingly may deepen our understanding of the wave-particle duality.

\section{ACKNOWLEDGMENTS}

This research was supported by the National Basic Research Program of China (Grants No. 2011CB922203, No. 2010CB923102, and No. 2012CB921603), the national Natural Science Foundation of China (Grants No. 11174026, No. 11274212, and No. 11174118), and the Natural Science Foundation of Jiangxi Province under Grant No. 20114BAB212003. The research of M.S.Z. is supported by an NPRP grant (Grant No. 4-346-1-061) from the Qatar National Research Fund.
[1] N. Bohr, Naturwissenschaften 16, 245 (1928).

[2] M. O. Scully, B. G. Englert, and H. Walther, Nature 351, 111 (1991); B. G. Englert, M. O. Scully, and H. Walther, ibid. 375, 367 (1995).

[3] E. P. Storey, S. M. Tan, M. J. Collett, and D. F. Walls, Nature 367, 626 (1994); 375, 368 (1995).

[4] R. P. Feynman, R. Leighton, and M. Sands, in The Feynman Lectures on Physics (Addison Wesley, Reading, MA, 1965), Vol. III.

[5] R. J. Glauber, Ann. N. Y. Acad. Sci. 480, 336 (1986).

[6] W. K. Wootters and W. H. Zurek, Phys. Rev. D 19, 473 (1979).

[7] D. M. Greenberger and A. Yasin, Phys. Lett. A 128, 391 (1988).

[8] G. Jaeger, A. Shimony, and L. Vaidman, Phys. Rev. A 51, 54 (1995).

[9] B. G. Englert, Phys. Rev. Lett. 77, 2154 (1996).

[10] H. Y. Liu, J. H. Huang, J. R. Gao, M. S. Zubairy, and S. Y. Zhu, Phys. Rev. A 85, 022106 (2012).
[11] T. Pfau, S. Spälter, Ch. Kurtsiefer, C. R. Ekstrom, and J. Mlynek, Phys. Rev. Lett. 73, 1223 (1994); M. S. Chapman, T. D. Hammond, A. Lenef, J. Schmiedmayer, R. A. Rubenstein, E. Smith, and D. E. Pritchard, ibid. 75, 3783 (1995); E. Buks, R. Schuster, M. Heiblum, D. Mahalu, and V. Umansky, Nature 391, 871 (1998); S. Dürr, T. Nonn, and G. Rempe, ibid. 395, 33 (1998); P. Bertet, S. Osnaghi, A. Rauschenbeutel, G. Nogues, A. Auffeves, M. Brune, J. M. Raimond, and S. Haroche, ibid. 411, 166 (2001).

[12] J. A. Wheeler, in Quantum Theory and Measurement, edited by J. A. Wheeler and W. H. Zurek (Princeton University Press, Princeton, NJ, 1984), pp. 182-213.

[13] V. Jacques, E. Wu, F. Grosshans, F. Treussart, P. Grangier, A. Aspect, and J.-F. Roch, Science 315, 966 (2007).

[14] V. Jacques, E. Wu, F. Grosshans, F. Treussart, P. Grangier, A. Aspect, and J.-F. Roch, Phys. Rev. Lett. 100, 220402 (2008). 
[15] R. Ionicioiu and D. R. Terno, Phys. Rev. Lett. 107, 230406 (2011).

[16] M. Schirber, Physics 4, 102 (2011).

[17] J. S. Tang, Y. L. Li, X. Y. Xu, G. Y. Xiang, C. F. Li, and G. C. Guo, Nat. Photonics 6, 602 (2012).

[18] A. Peruzzo, P. Shadbolt, N. Brunner, S. Popescu, and J. L. O'Brien, Science 338, 634 (2012).

[19] F. Kaiser, T. Coudreau, P. Milman, D. B. Ostrowsky, and S. Tanzilli, Science 338, 637 (2012).

[20] J. H. Huang, S. Wölk, S. Y. Zhu, and M. S. Zubairy, Phys. Rev. A 87, 022107 (2013).
[21] G. Björk, J. Söderholm, A. Trifonov, T. Tsegaye, and A. Karlsson, Phys. Rev. A 60, 1874 (1999); P. Busch and C. Shilladay, Phys. Rep. 435, 1 (2006).

[22] P. G. Kwiat, K. Mattle, H. Weinfurter, A. Zeilinger, A. V. Sergienko, and Y. Shih, Phys. Rev. Lett. 75, 4337 (1995); C. Kurtsiefer, M. Oberparleiter, and H. Weinfurter, Phys. Rev. A 64, 023802 (2001); R. Horn, P. Abolghasem, B. J. Bijlani, D. Kang, A. S. Helmy, and G. Weihs, Phys. Rev. Lett. 108, 153605 (2012).

[23] C. K. Hong, Z. Y. Ou, and L. Mandel, Phys. Rev. Lett. 59, 2044 (1987). 